\title{
Numerical modelling of colloidal fluid in a viscous micropump
}

\author{
H. El-Sadi \& N. Esmail \\ Department of Mechanical and Industrial Engineering, \\ Concordia University, Quebec, Canada
}

\begin{abstract}
Non-Newtonian fluid can be encountered in many applications of Microdevices. In this study, two-dimensional non-Newtonian simulations of a viscous micropump were performed. The viscous micropump consists of a rotating cylinder located eccentrically inside a microchannel. When the cylinder rotates, a net force is transferred to the fluid due to the unequal shear stresses on the upper and lower surfaces of the cylinder, thus causing the fluid to displace. Non-Newtonian fluid is predicted by Navier Stokes equations and proposed by a modified Bingham model to describe the fluid flow

Keywords: micropump, non-Newtonian, Bingham model, eccentricity, bulk velocity, microchannel, Navier Stokes equation, shear stress.
\end{abstract}

\section{Introduction}

Increasing efforts are being directed towards applying the technologies of microfluidic, to the development of micro-devices for a wide range of applications such as medical, biological and related technologies. The main advantage of MEMS, in addition to their small size, is the fact that the manufacturing costs are remarkably lower when compared to their bigger counterparts, due to the mass fabrication methods used to produce them.

Micropumps are between the most developed of all MEMS devices, and have been executed into the mainstream (Voigt et al [1], Schomburg and Goll [2]). Micropumps are imperative components for distributing fluid and samples in microanalysis system. Positive displacement pumping is the most widespread method used in micropumps, on the other hand the actuation of the reciprocating diaphragm can be achieved by different principles such as piezoelectric, 
pneumatic, electrostatic etc. (Shoji and Esashi [3], Gravesen et al [4]). However, various pumping ideas were proposed to overcome the valve problem correlated with positive displacement pumps.

Our interests in this work are to study the effect of the height of viscous micropump and dynamic parameters on the flow behavior of colloidal liquid in a viscous micropump.

\section{Problem description}

\subsection{Pump geometry}

The pump geometry is shown in figure 1. The dimensionless parameters, which are associated to the geometry, are channel height (S) and cylinder eccentricity (ع), defined as:

$$
\begin{gathered}
S=\frac{h}{d} \\
\varepsilon=\frac{\left(h-2 Y_{c}\right)}{h-d}
\end{gathered}
$$

Where $\mathrm{d}$ is the cylinder diameter and $\mathrm{h}$ is the channel height, $Y_{c}$ the distance between the lower wall of the channel and the center of the cylinder. The cylinder rotates with an angular velocity $\omega$ and is placed at different positions between the upper and lower plates of the channel based on the eccentricity. The pressure is exerted on the inlet and outlet of the channel.

\subsection{Mathematical equations and boundary conditions}

For incompressible and steady state non-Newtonian fluid, the continuity equation and the equation of motion are:

$$
\begin{gathered}
\nabla \cdot V=0 \\
(V . \nabla V)+\nabla p+\nabla \tau=0
\end{gathered}
$$

with:

$$
\nabla \tau=-\eta \nabla \cdot \dot{\gamma} \text { and } \dot{\gamma}=\nabla V+(\nabla V)^{T}
$$

where $\tau$ is the extra stress tensor, $\dot{\gamma}$ the rate of strain tensor, $\mathrm{V}$ the tangential velocity and $\eta$ the fluid viscosity. Since non-Newtonian fluid respond to the imposed flows. A different constitutive equation is needed, material $\tau=f(\nabla V, V$, information $)$.

\section{Results and discussion}

The rotor eccentricity has a crucial effect on the performance of micro viscous pump. For colloidal fluid, the shear stress increases with increasing the rate of strain. Figures 3 shows the curves $\tau=f(\varepsilon)$ respectively, where $\mathrm{S}$ (channel height) is maintained constant. It is concluded that the pumping performance of colloidal fluid is decreasing with the channel height. 
$\mathbf{L}$

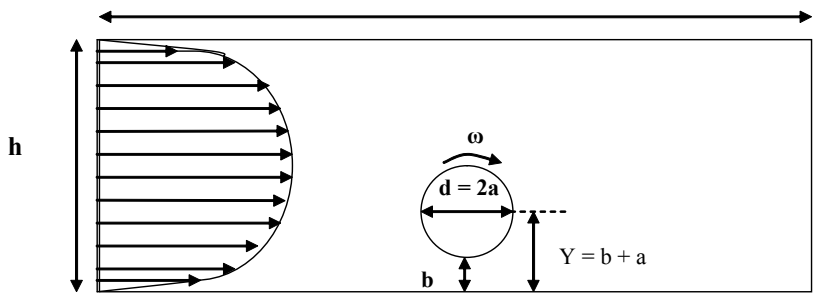

Figure 1: $\quad$ Problem geometry and variable velocity distribution.

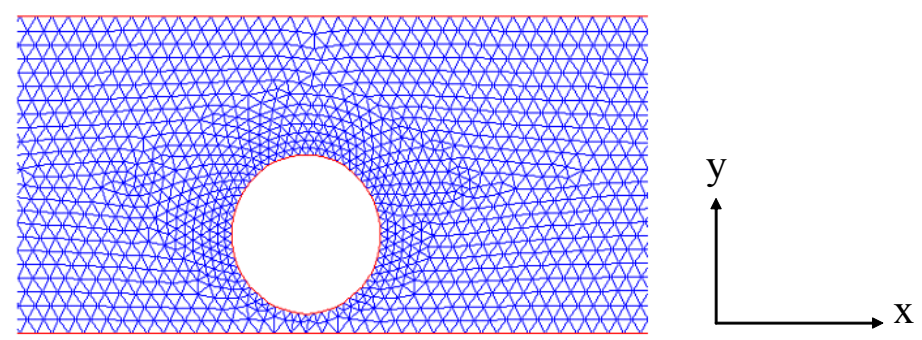

Figure 2: An example of mesh generation.

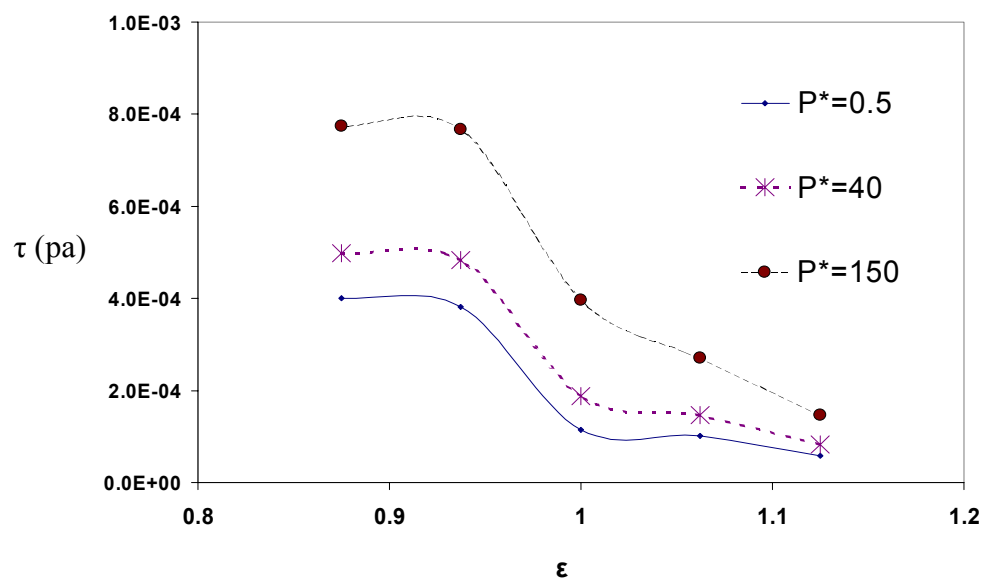

Figure 3: Changes of the stress as a function of eccentricity. For $\mathrm{p}^{*}=0.5,40$ and 150 . 


\section{References}

[1] Voigt P. Schrag G. and Wachutka G. "Electrofluidic full-system modeling of a flap valve micropump based on Kirchhoffian network theory, sensors and actuators" A66, 9-14(1998).

[2] Schomburg W. K. and Goll C. "Design optimization of bistable microdiaphragm valves, sensors and actuators" A 64,259-264 (1998).

[3] Shoji S. and Esashi M., "Microflow devices and systems", J. Micromech. Microeng. 4, 157(1994).

[4] Gravesen P., Branebjerg J. and Jensen O.S., "Microfluidics-a Review", J. Micromech. Microeng. 3, 168-182 (1993). 